\title{
Performance Analysis of MIMO OFDM System by using LS and MMSE Techniques
}

\author{
Deepika Jharbade \\ Department of Electrical and Electronics \\ Institute Of Engineering and Technology, DAVV \\ Indore
}

\author{
Madhvi Jangalwa \\ Department of Electrical and Electronics \\ Institute Of Engineering and Technology, DAVV \\ Indore
}

\begin{abstract}
In today's world, high speed is an important factor to be considered in wireless communication systems. This is because high speed is required for access of video, images, and many others multimedia application. One way to achieve high data rate is OFDM. Orthogonal Frequency Division Multiplexing (OFDM) is a technique which transmitted data parallel and this data are orthogonal to each other, hence result high data rate in wireless transmission as large no. of data is transmitted at same time. Another way is MIMO system. To increase the diversity gain or to enhance the system capacity on time-variant and frequency-selective channels, OFDM may be combining with multiple transmitter antenna and receiver antenna, this resulting MIMO OFDM system. MIMO-OFDM, a new wireless $4 \mathrm{G}$ technologies, has capability of enhance data rate transmission and robustness against multi-path fading. In this paper, two techniques Least Square (LS) and Minimum Mean Square Error (MMSE) are used to analysis the performance of MIMO OFDM System also used to reduce BER.
\end{abstract}

\section{Keywords}

MIMO-OFDM system, Diversity gain, LS techniques, MMSE techniques, multipath fading.

\section{INTRODUCTION}

All researcher and engineers are always tried to improve speed in wireless communication. We had seen that there are so many developments in wireless communication. The first generation $(1 \mathrm{G})$ of analog mobile communication system can only provide voice service. The second generation (2G) digital mobile communication system has better performance, but the bandwidth is restricted [1]. The $3 \mathrm{G}$ system can enhance the data rate and cover the whole earth. But with the continuous development of data, images, video, and other multimedia applications, the available data rate will be quickly saturate. This increases the demand of bandwidth. In view of the shortage of bandwidth spectrum nowadays, in order to transmit data with high speed and high capacity, the wireless communication should have very high spectrum efficiency and the capacity of overcoming the channel fading in the environment of multi-path channel. It is very difficult to match these requests for the traditional modulation technique, but the MIMO-OFDM system can meet the requirements.
Orthogonal Frequency Division Multiplexing (OFDM) is a multi-carrier transmission technique proposed in mid 1960's. In frequency selective channel, delay spread of the channel impulse response introduces inter-symbol interference (ISI) in a single carrier system, which causes severe system performance degradation if not taken care of. OFDM effectively counters the channel delay spread by converting channel into a number of overlapping but mutually orthogonal sub-channels in frequency domain. By sending information in parallel with larger symbol durations, OFDM systems avoid the ISI significantly [2-5] and increase data rate. The data rate can be further increased via the exploitation of the MIMO technique. MIMO offers additional parallel channels in spatial domain to boost the data rate. Hence, MIMO-OFDM is a promising combination for the high data requirement of future wireless systems $[6,7]$.

The major challenge faced in MIMO-OFDM system is how to obtain the channel state information accurately for detection of information. The channel state information can be achieved through training based estimation, blind based estimation and semi blind based channel Estimation. The blind channel estimation is carried out by evaluating the statistical information of the channel and certain properties of the transmitted signals [8, 9]. Blind Channel Estimation has its advantage in that it has no overhead loss; it is only applicable to slowly time-varying channels due to its need for a long data record. In training based channel estimation algorithms, training symbols or pilot tones that are known $a$ priori to the receiver, are multiplexed along with the data stream for channel estimation [10]. Semi-blind channel technique is hybrid of blind and training technique, utilizing pilots and other natural constraints to perform channel estimation [11].

The training-based method channel estimation can be performed by either block type or by comb pilots. The block type pilot channel estimation, has been developed under the assumption of slow fading channel; this assumes that the channel transfer function is not changing very rapidly it can be constant over transmission of few OFDM symbols. The comb-type pilot channel estimation has been introduced in case where the channel changes even in one OFDM block. The signal is transformed into a linear form which is suitable for LS and MMSE estimation algorithm. MMSE shown much better performance than LS but it is more complex to estimate CIR. 
The organization of the paper is as follows. Section 2 describe MIMO OFDM system model. Section 3 provided the channel estimation algorithm for MIMO-OFDM and section 4 contains the conclusion and results.

\section{MIMO OFDM SYSTEM MODEL}

MIMO OFDM is a $4 \mathrm{G}$ system model. It is used to enhanced the data rate transmission between transmitter and receiver.

\subsection{MIMO OFDM Transmitter System}

Fig1 shows block diagram of MIMO OFDM System. The binary input data is first modulated different modulation technique such as QPSK, BPSK, depending upon the channel like fading channel, IEEE 802.11.After this coding is done. The serial data is fed to serial to parallel converter $[12,13]$. This reduce data rate by $\mathrm{N}$ times, where $\mathrm{N}$ is number of parallel stream. Each parallel stream acquires small bandwidth in spectrum. Then, inserting pilots signal either to all subcarrier with specific period of blocks or within uniform period of frequency in all blocks. Then this signal is given to IFFT block. The cyclic prefix are added to the signal to prevent inter symbol interference. Finally, these signals are transmit with $\mathrm{M}_{\mathrm{t}}$ no. of transmitter.

transmitted signal:

$$
X(n)=\sum_{i=0}^{N_{i}-1} x_{i} \exp (j 2 \pi n / N)
$$

where $\mathrm{x}_{\mathrm{i}}$ is input signal.

Space Time Block coding are used to transmitted data from no. of transmitted antennas. Space-time-block-codes can be view as repetition code over space and time, simultaneously transmitting the same data over different antennas. The STBC achieve significant error rate improvements over singleantenna system. Their original scheme was based on trellis codes but the simpler block codes were utilized by S.Alamouti.

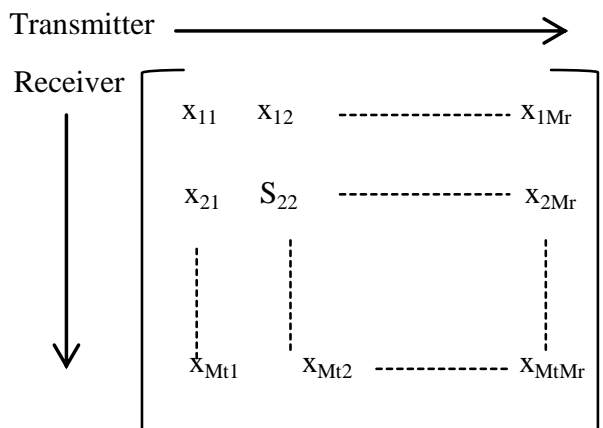

Alamouti's code uses a complex orthogonal design, in which the transmission matrix is square and satisfies the condition for complex orthogonality in both space and time dimension. It is the only orthogonal STBC that achieves rate-1, i.e it is

The received signal is given by

$$
\mathrm{N}-1
$$

$Y_{i}(n)=\underset{n=0}{\sum} H_{i j} X_{j}(n)+n$ only STBC that can achieve its full diversity gain without needing to sacrifices its data rate. This code is designed for two transmit and one receive antenna and the code of matrix is given as

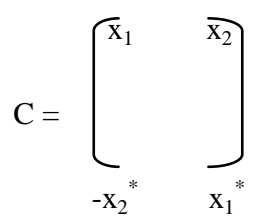

Alamouti's scheme is that the sequence transmitted from the different antennas is orthogonal since the matrix of $\mathrm{S}$ times the Hermitian matrix $\mathrm{S}$ is equal to the identity matrix such as:

$$
\mathrm{C}^{*} \mathrm{C}^{\mathrm{H}}=\left[\begin{array}{cc}
\mathrm{x}_{1} & \mathrm{x}_{2} \\
-\mathrm{x}_{1}{ }^{*} & \mathrm{x}_{2}{ }^{*} \\
\left|\mathrm{x}_{0}\right|^{2} & \left|\mathrm{x}_{1}\right|^{2} \mathrm{I}
\end{array}\right]\left[\begin{array}{cc}
\mathrm{x}_{1}{ }^{*} & -\mathrm{x}_{2} \\
\mathrm{x}_{1}{ }^{*} & \mathrm{x}_{2}
\end{array}\right]
$$

Where the superscript ${ }^{\mathrm{H}}$ represents the Hermitian matrix of S which is the transpose and conjugate of the matrix S and I is a $2 \times 2$ identity matrix. At the receiver, the received signals at time $t$ and $t+T$ can be expressed as in (1). The received signal will be denoted by $r_{1}$ and $r_{2}$ at time $t$ and $t+T$ respectively.

$$
\begin{aligned}
& \mathrm{Y}_{1}=\mathrm{H}_{1} \mathrm{x}_{1}+\mathrm{H}_{2} \mathrm{x}_{2}+\mathrm{n}_{0} \\
& \mathrm{Y}_{2}=-\mathrm{H}_{1} \mathrm{x}_{2}{ }^{*}+\mathrm{H}_{2} \mathrm{x}_{1}{ }^{*}+\mathrm{n}_{1}
\end{aligned}
$$

Where $\mathrm{n}_{1}$ and $\mathrm{n}_{2}$ represent the white Gaussian noise samples. Originally, STBC channel parameters were assumed known at the receiver. Therefore, transmitted symbols $\mathrm{s}_{0}$ and $\mathrm{s}_{1}$ can be recovered by combining the received signal $r_{1}$ and $r_{2}$ as:

$$
\begin{aligned}
& \mathrm{x}_{1}=\mathrm{Y}_{1} \mathrm{H}_{1}{ }^{*+} \mathrm{Y}_{2}{ }^{*} \mathrm{H}_{2} \\
& \mathrm{x}_{2}=\mathrm{H}_{2}{ }^{*} \mathrm{Y}_{1}-\mathrm{H}_{1} \mathrm{Y}_{2}{ }^{*}
\end{aligned}
$$

from (2) and (3), and due to the orthogonality of the transmitted matrix, recover of $s_{1}$ and $s_{2}$ is possible.

\subsection{MIMO OFDM Receiver System}

Let, $M_{r}$ be no. of receiver antenna which receives the $M_{t}$ transmitted signal. $\mathrm{Y}_{\mathrm{i}}(\mathrm{n})$ signal is received at the receiver. Received signal is combination of transmitted signal $X_{j}(n)$ and noise added during transmission. At receiver side, firstly cyclic prefix are remove. After removed cyclic prefix FFT is taken. By using pilot signal, channel impulse response is estimated. CIR can be estimated either by LS technique or by MMSE technique fig2.

where $Y_{i}(n)=\left[\begin{array}{lll}Y_{i}\left(n_{1}\right), \quad \ldots & Y_{i}\left(n_{M r}\right)\end{array}\right]^{T}$ and $X_{j}(n)=\left[X_{j}\left(n_{1}\right), \ldots\right.$ $\left.X_{j}\left(n_{M t}\right)\right]^{T}$ are vectors with the length $M . H_{i}, j$ is $L \times 1$ vector from jth transmit antenna to ith receive antenna. $\mathrm{L}$ is maximum length of channel. $\mathrm{n}$ is $\mathrm{M} \times 1$ additive white Gaussian noise vector. 


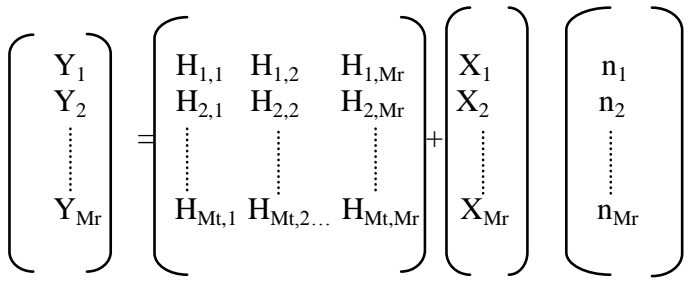

\section{LS AND MMSE TECHNIQUE OF MIMO OFDM SYSTEM}

LS and MMSE are techniques which are used to receiver multiple signals in MIMO OFDM system. These techniques are based on the principle of pilot-symbol channel estimation. The pilot symbols have been inserted into the signal by two different approaches, as shown in fig3.

In a block-type (BT) block type pilot arrangement shown in fig3 (a), where pilot tones are inserted into all frequency bins within periodic intervals of OFDM blocks, which is transmitted regularly after a fixed number of data symbols, $N \mathrm{D}$. In a comb-type (CT) pilot sub-carrier arrangement shown in fig3 (b), pilot tones are inserted into each OFDM symbol symbols with a specific period of frequency bins [14].

For comb-type pilot sub-carrier arrangement, let $\mathrm{Np}$ pilot signals $\mathrm{Xp}(\mathrm{m}) ; \mathrm{m}=0 ; 1 ;::: ; \mathrm{Np}-1$, are uniformly inserted into $\mathrm{X}(\mathrm{k})$. That is, the total $\mathrm{N}$ sub-carriers are divided into $\mathrm{Np}$ groups, each with $\mathrm{L}=\mathrm{N}=\mathrm{Np}$ adjacent sub-carriers. In each group, the first sub-carrier is used to transmit pilot signal.

Assuming training over $\mathrm{k}$ consecutive OFDM symbol, the sequence (1) can be written as

$\mathrm{Y}_{\mathrm{p}}=\mathrm{X}_{\mathrm{p}} \mathrm{H}_{\mathrm{p}}+\mathrm{n}_{\mathrm{p}}$

Where $n_{p}$ is the vector of Gaussian noise in pilot subcarriers.

\subsection{LS Estimation Technique}

MIMO OFDM significantly simplifies baseband receiver processing, by eliminating the need for a complex MIMO Equalizer. $Y_{1}, Y_{2} \ldots Y_{n}$ can be simply processed by MIMO LS which is nothing but a Zeroforcing receiver and MIMO MMSE for detection of the $\mathrm{X}_{1}, \mathrm{X}_{2} \ldots \mathrm{X}_{\mathrm{Mt}}$ In LS and MMSE MIMO technique we assume that channel matrix is known at receiver. Than $\mathrm{X}_{\mathrm{i}}(\mathrm{n})$ evaluate by using $\mathrm{LS}$ technique is

$$
X_{j}(n)=H_{i j}(n)^{-1} Y_{i}(n)
$$

\subsection{MMSE Estimation Technique}

$\mathrm{X}_{\mathrm{j}}(\mathrm{n})_{\text {MMSE }}=\operatorname{inv}\left[\mathrm{H}_{\mathrm{ij}}(\mathrm{n})^{\mathrm{H}} * \mathrm{H}_{\mathrm{ij}}(\mathrm{n})+\sigma_{0} \mathrm{I}(\mathrm{n})\right]^{*} \mathrm{H}_{\mathrm{ij}}(\mathrm{n})^{\mathrm{H}} * \mathrm{Y}_{\mathrm{i}}(\mathrm{n})$

Where $\mathrm{H}^{\mathrm{H}}$ is hermitan matrix and $\sigma_{0}$ is assume to be one. MMSE is typical to calculate because it required too much calculation

\section{CONCLUSION}

The aim of this work presented here was to analysis the performance of transmitted signal by combining OFDM with MIMO system. This focuses on to reduce BER via using various parameters. Two algorithm LS and MMSE are used here. Fig.4 shows graph of OFDM system over AWGN and Rayleigh channel. AWGN shows good result than Rayleigh channel. BER of AWGN channel is increased by $10 \%$ as compare to Rayleigh channel at $4 \mathrm{db}$. Graph of MIMO OFDM system for different no of receiver is seen in Fig.5. BER of $2 \times 4$ at $4 \mathrm{db}$ MIMO OFDM system is increased by $33 \%$ when compare to 2x1.Fig.6 and Fig.7 shows simulation result for $2 \times 1,2 \times 2$ antennas respectively. In this different modulation technique are compared. Figure conclude that BPSK show better BER compare to QPSK and QAM. Fig.8 compares the OFDM technique and MIMO OFDM technique over the AWGN channel. It shows that if multiple antenna combine with OFDM technique, it show better BER than the OFDM technique. BER Of MIMO OFDM increased by $30 \%$ at 4db.Result of LS and MMSE is evaluated in Fig.9. Graph concludes that MMSE has good BER than LS. BER of MMSE is increased by $42 \%$ at $4 \mathrm{db}$. There is a comparison between OFDM for $1 / 8$ and $1 / 4$ cyclic prefix, shown in Fig.10. Graph conclude that $1 / 8$ cyclic prefix show better BER than $1 / 4$ cyclic prefix. Similarly Fig. 11 show graph for MMSE cyclic prefix. 1/8 cyclic prefix BER is better than $1 / 4$ cyclic prefix.

Table1. Simulation Parameter

\begin{tabular}{|l|l|}
\hline Parameter & Value \\
\hline Channel coding & Convolution coding \\
\hline Modulation & BPSK,QPSK,qam \\
\hline FFT size & 256 \\
\hline Channel & Rayleigh, AWGN \\
\hline Cyclic prefix & $1 / 8,1 / 4$ \\
\hline No.of Transmitter & 1,2 \\
\hline No. of Receiver & $1,2,4$ \\
\hline
\end{tabular}




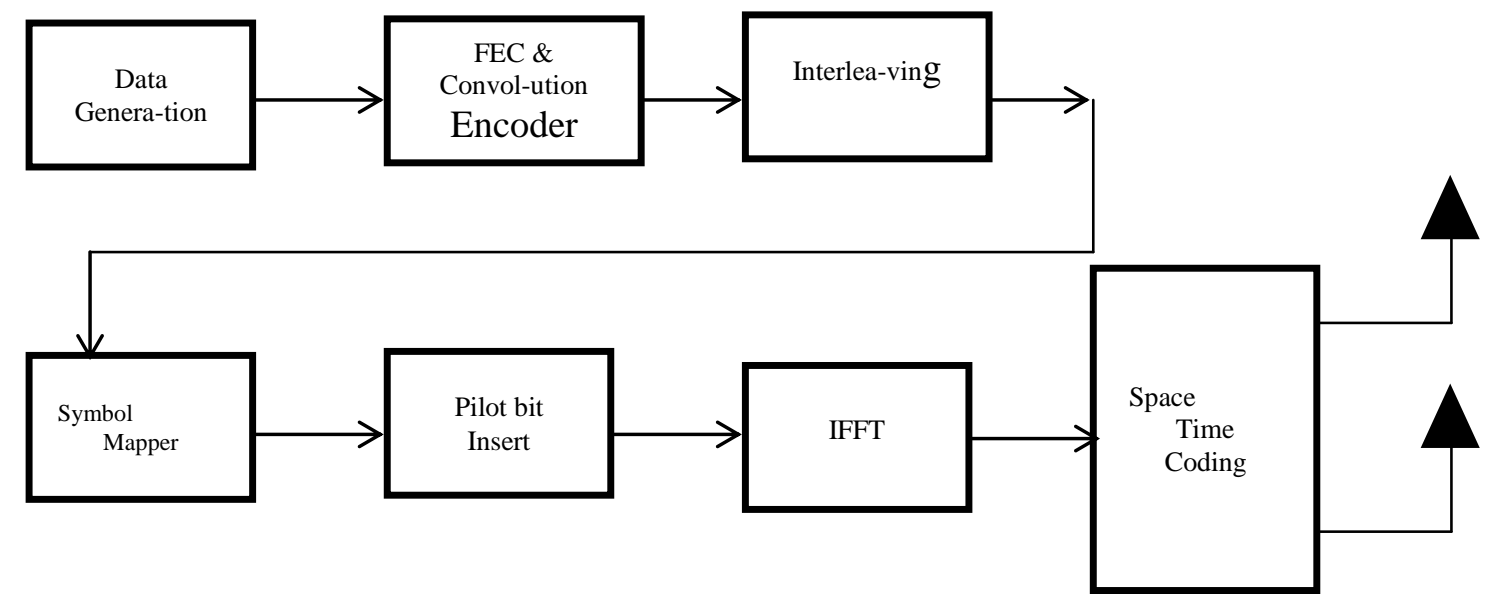

Fig1: MIMO OFDM transmitter model

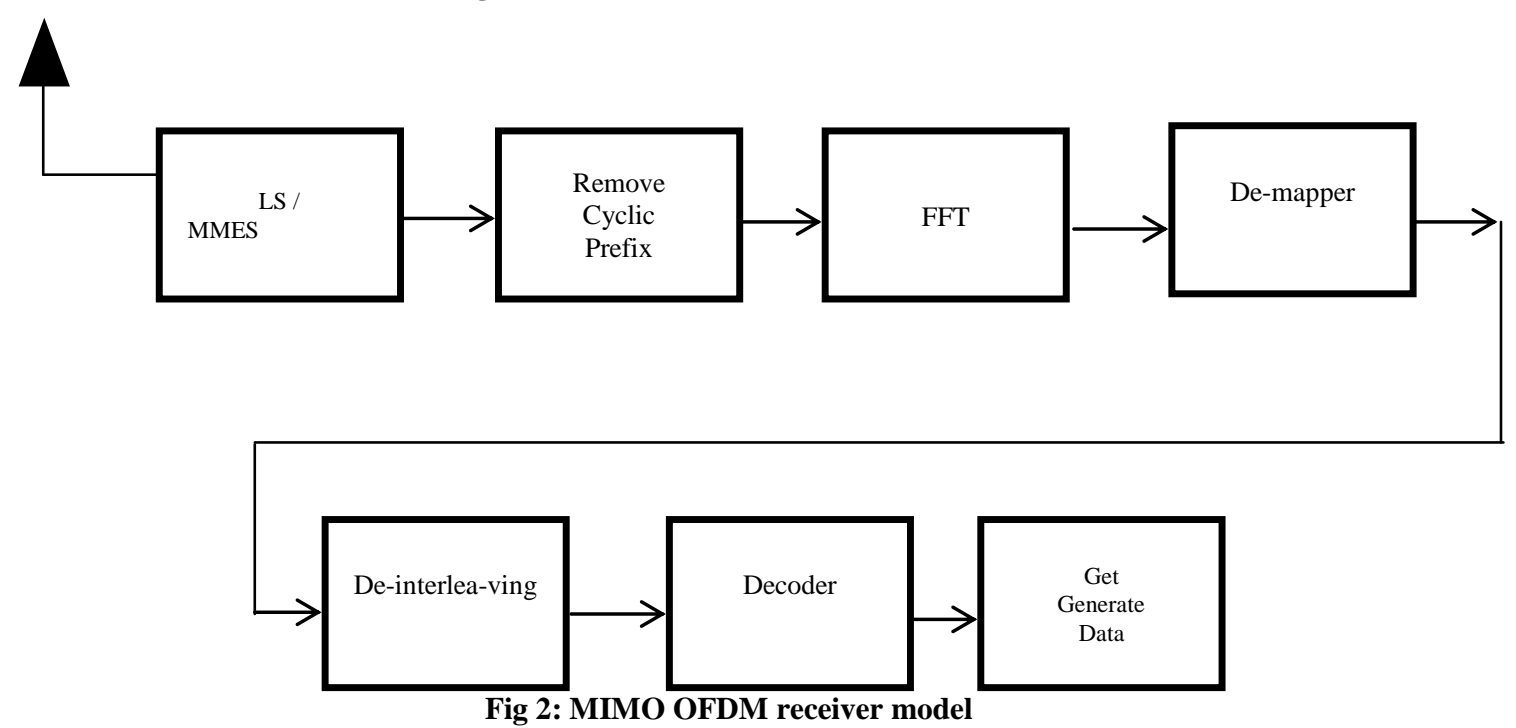

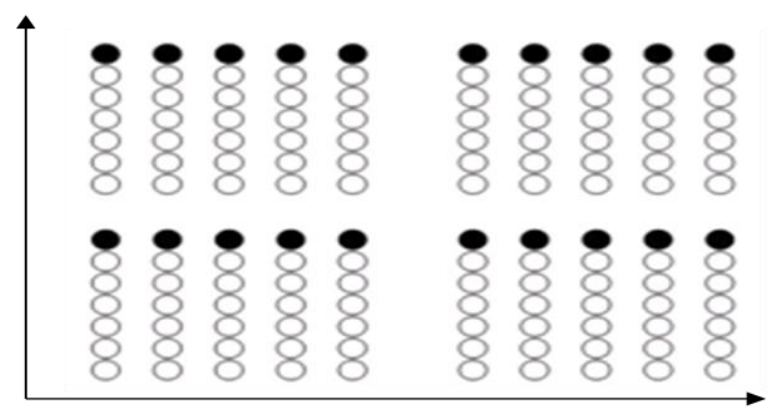

(a) block type

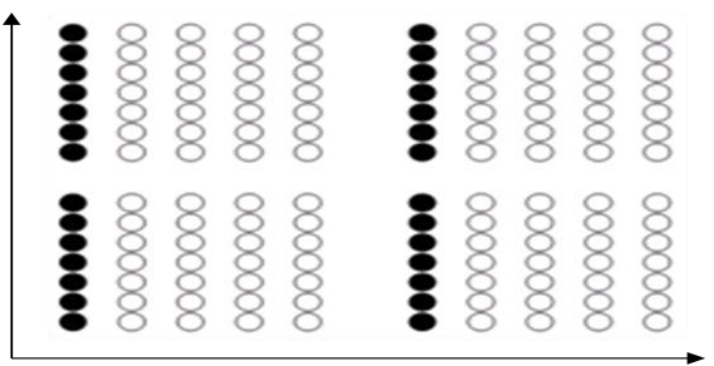

(b) comp type

Fig3: How Pilot signal can be inserted during transmission 


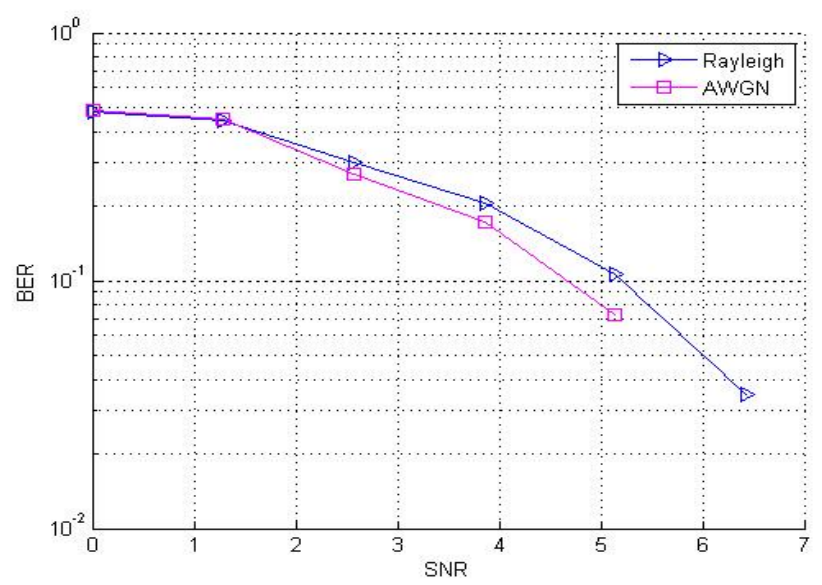

Fig4: Performance of OFDM system over AWGN and Rayleigh channel

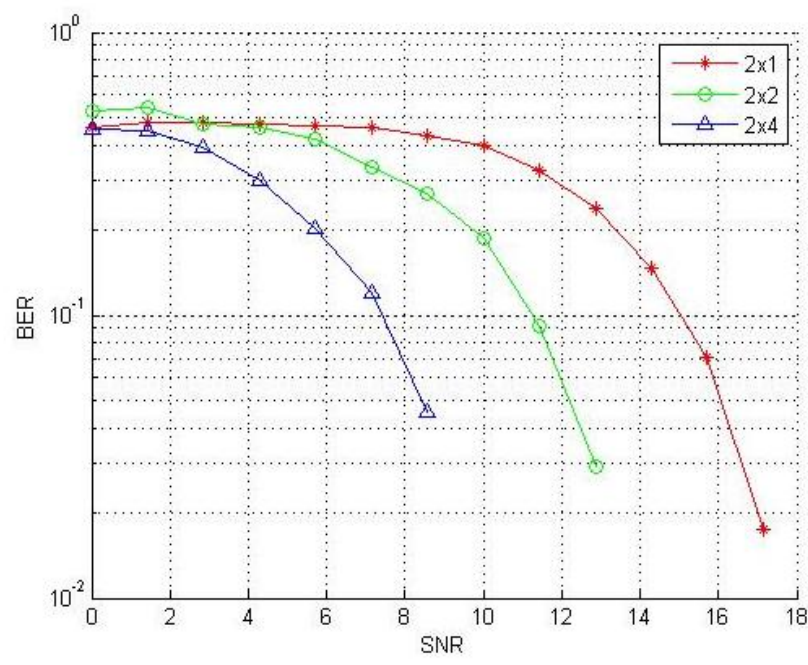

Fig5: Comparison of MIMO OFDM for different no. of Receiver

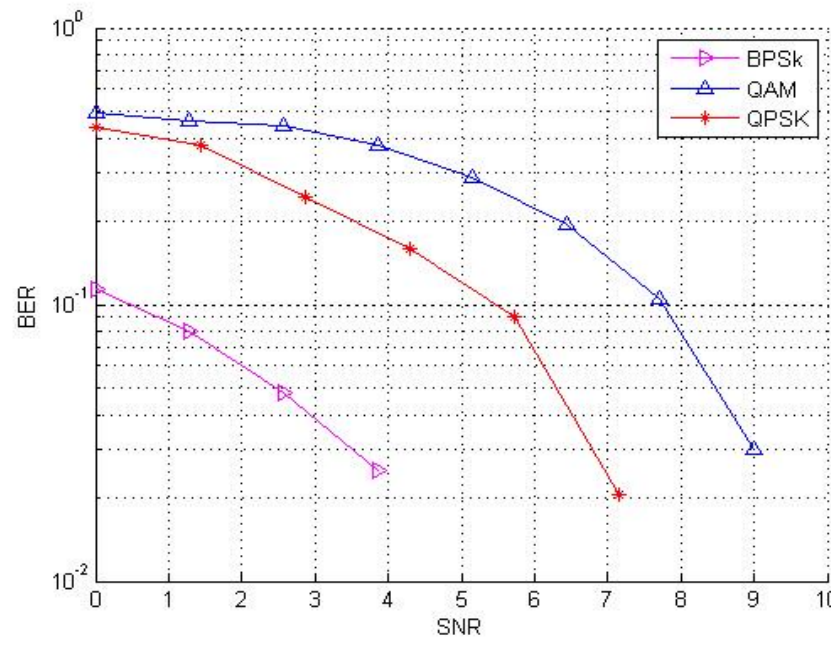

Fig6: Comparison between different modulation techniques for 2x1 MIMO OFDM systems

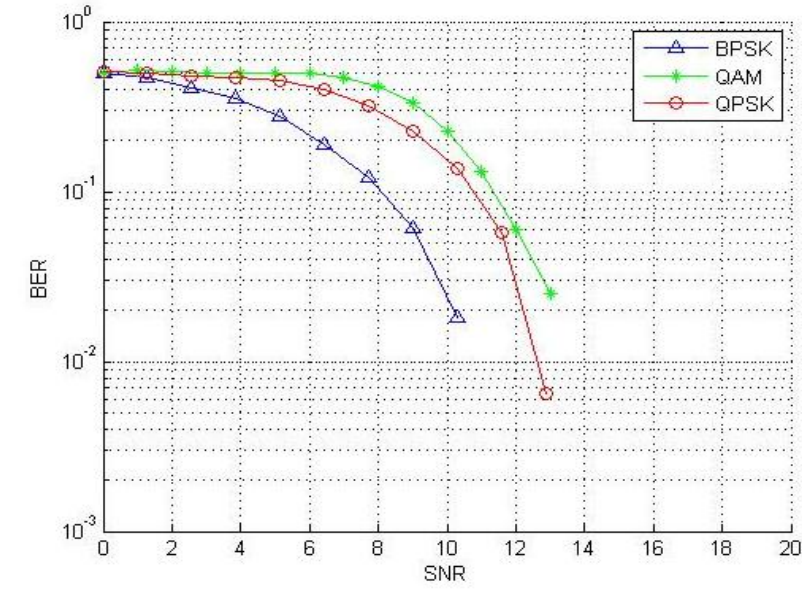

Fig7: Comparison between different modulation techniques for 2x2 MIMO OFDM systems

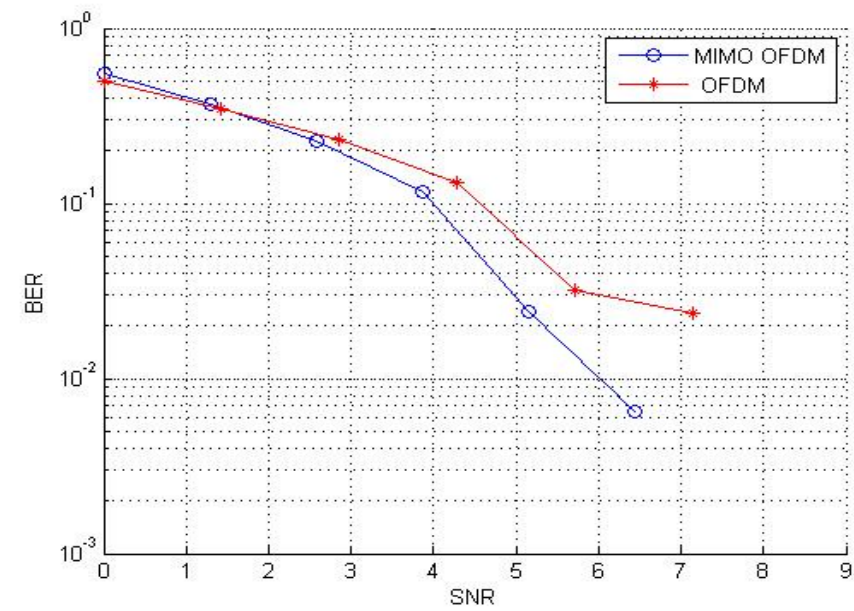

Fig8: Performance of OFDM and MIMO OFDM over AWGN channel

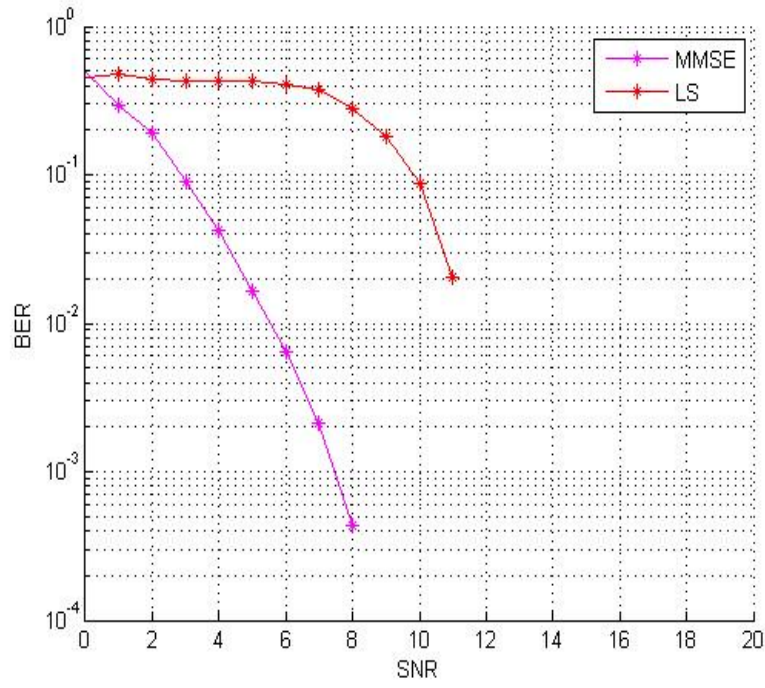

Fig9: Comparison between LS and MMSE channel estimation technique for MIMO OFDM system. 


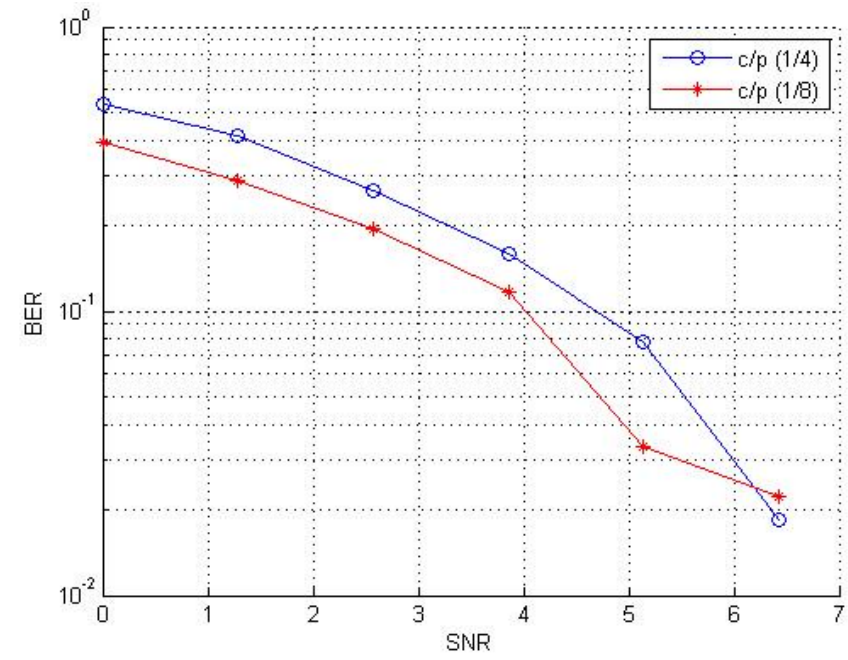

Fig1:0 OFDM system for different cyclic prefix 1/4 and 1/8

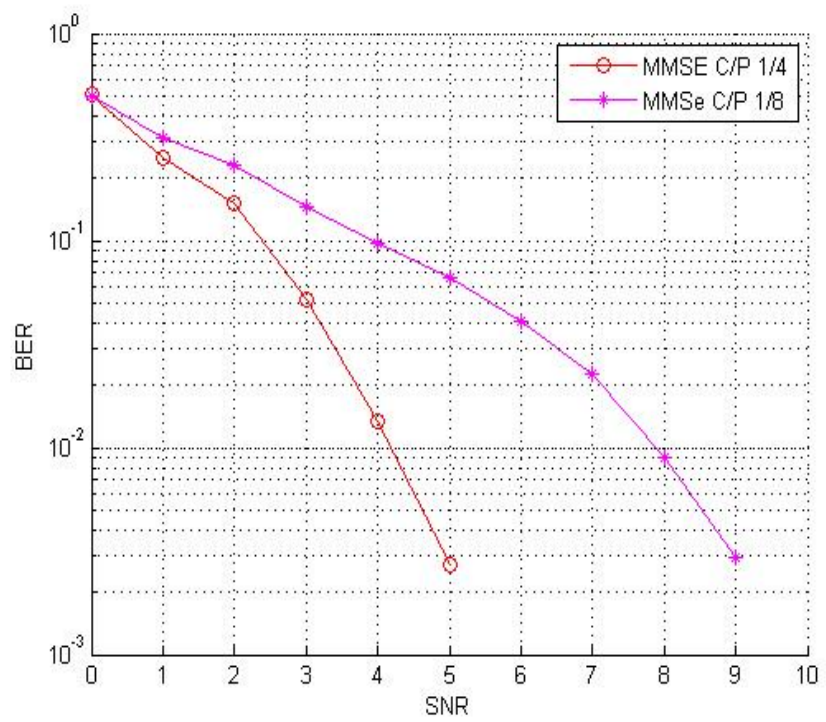

Fig11: MMSE receiver for different cyclic prefix 1/4 and $1 / 8$

\section{ACKNOWLEDGMENTS}

Our thanks to those people who directly or indirectly help to doing this work.

\section{REFERENCES}

[1] Wang, Xiaodang, "OFDM and its application to $4 \mathrm{G}^{\prime \prime}, 14$ th annual conference on wireless and optical communications, USA, pp. 69-71, 2005.
[2] L.J cimini, "Analysis and simulation of digital mobile channel using OFDM", IEEE trans. Comm.. vol 33, no.7, pp 665-675 june 1980.

[3] H.Boelcskie \& D.Gesbert \& A.J Paulraj, "ON the capacity of OFDM based Spatial multiplexing System", IEEE trans. Comm.. vol.50 no.2, pp 225-234, Feb 2002.

[4] O. Edfors, M. Sandell, J.-J. van de Beek, S. K. Wilson, and P. O. Borjesson, "OFDM channel estimation by singular value decomposition", IEEE Trans. Commun., 46(7), pp- 931-939, July 1998.

[5] Z. Guo and W. Zhu. 2-D, " channel estimation for OFDM/SDMA", In Proc. IEEE World Wireless Congress, San Francisco, CA, May 2002.

[6] J. Xuehua, C. Peijiang, "Study and Implementation of MIMO OFDM System Based on Matlab", International Conference on Information Technology and Computer Science, pp 554-557, 2009.

[7] G.L Studer J.R Barry, S.W.Mclaughlin,Ye Li,M.A. Ingram and T.G Pratt," Broadband MIMO-OFDM wireless communication", proceedings of IEEE, vol. 92, no.2, pp 271-294,Feb 2004.

[8] A. Petropulu, R. Zhang, and R. Lin, "Blind OFDM channel estimation through simple linear pre-coding", IEEE Transactions on Wireless Communications, vol. 3, no.2,March 2004, pp. 647-655.

[9] A. Zaier1 and R. Bouallègue "Blind channel estimation enhancement for mimo- ofdm systems under high mobility conditions " International Journal of Wireless \& Mobile Networks(IJWMN) Vol. 4, No.1, February 2012.

[10]O. Simeone, Y. Bar-Ness, U. Spagnolini, "Pilot Based Channel Estimation for OFDM SystemsbyTrackingthe Delay Subspace", IEEE Transactions on Wireless Communications, Vol. 3, No.1, January 2004.

[11]A.K. Jagannatham, C.R. Murthy and B.D. Rao" A semiblind MIMO channel estimation scheme for MRT'IEEE, pp $585-588,2005$

[12] H.Minn, Member, IEEE and N.Al-Dhahir, Senior Member, IEEE, "Optimal Training Signals for MIMO OFDM Channel Estimation", pp 1-10, 2004.

[13] K. P.Bagadi, Prof. Susmita Das, " MIMO-OFDM Channel Estimation using Pilot Carries", International Journal of Computer Applications (0975 - 8887) Vol. 2 No.3,pp 81-88, May 2010.

[14] D Hu, L Yang, L He, Y Shi, " Optimal pilot sequence design for Multiple Input Multiple Output OFDM systems", IEEE Global Telecom Conference (GLOBECOM'05), (Saint Louis, USA, 2005), pp. 2260 2264. 